\title{
Ecotype-Dependent Response of Bacterial Communities Associated with Arabidopsis to Cold Acclimation
}

\author{
Mohammad Etemadi, Institute of Environmental Biotechnology, Graz University of Technology Graz, Austria; \\ Ellen Zuther, Max-Planck-Institut für Molekulare Pflanzenphysiologie, Am Mühlenberg 1, D-14476, Potsdam, \\ Germany; Henry Müller, Institute of Environmental Biotechnology, Graz University of Technology Graz, Austria; \\ Dirk K. Hincha, Max-Planck-Institut für Molekulare Pflanzenphysiologie, Am Mühlenberg 1, D-14476, Potsdam, \\ Germany; and Gabriele Berg, ${ }^{\dagger}$ Institute of Environmental Biotechnology, Graz University of Technology Graz, Austria
}

Accepted for publication 03 December 2017.

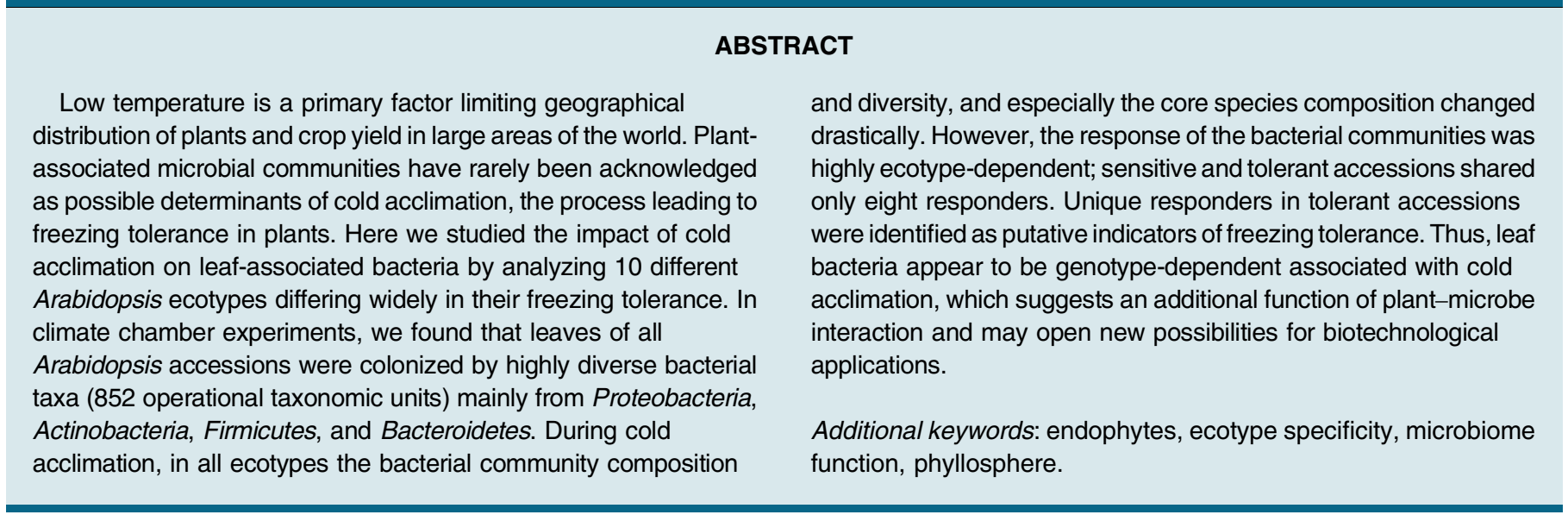

Periods of low and freezing temperatures are common major environmental challenges that plants from temperate regions face during their life cycle. They are a primary factor limiting the geographical distribution of native plant species but also the growth and yield of crops in many areas of the world (Xin and Browse 2000). In a process termed cold acclimation, plants from temperate climates increase their freezing tolerance during exposure to low, nonfreezing temperatures (Steponkus 1984). Natural variation of freezing tolerance along latitudinal climate gradients provides an excellent tool to study the potential importance of metabolic and physiological differences among accessions of a species for variation in freezing tolerance. Arabidopsis thaliana L. is a model species for temperate plants that is widely distributed on the Northern hemisphere. Natural accessions show different levels of freezing tolerance (Mckhann et al. 2008; Zhen and Ungerer 2008), and in a study with 54 accessions,

${ }^{\dagger}$ Corresponding author: G. Berg; E-mail: gabriele.berg@tugraz.at

*The $e$-Xtra logo stands for "electronic extra" and indicates that four supplementary figures and six supplementary tables are published online.

(C) 2018 The American Phytopathological Society evidence for latitudinal and longitudinal clines in nonacclimated and acclimated freezing tolerance were presented (Zuther et al. 2012). Massive changes in gene expression and content of primary metabolites (Guy et al. 2008; Hincha et al. 2012) as well as lipids and flavonoids (Degenkolbe 2012; Schulz et al. 2015) were identified during cold acclimation. As an example, expression of some CBFregulated $C O R$ genes and sugar content was correlated with freezing tolerance in the acclimated but not nonacclimated state in an analysis with 54 Arabidopsis accessions (Zuther et al. 2012). However, it is unclear whether these changes can quantitatively account for the observed differences in freezing tolerance among the accessions. Here, we have tested the hypothesis that the bacterial communities associated with Arabidopsis leaves are influenced by cold acclimation and differ between freezing tolerant and sensitive accessions. The microbiome of this plant species is well studied (Bai et al. 2015; Schlaeppi et al. 2014) and has previously been used to explore the role of microbiomes in various other processes, e.g., in the early flowering effect (Panke-Buisse et al. 2017).

The plant microbiota has been known as one of the key determinants of plant growth and health since more than 100 years (Hartmann et al. 2007; Weller et al. 2002). Recently, much deeper insights into the function of plant-associated microbial communities 
were gained by omics technologies (Bulgarelli et al. 2012; Mendes et al. 2011). The most recent research has shown that the microbiome is not only involved in coping with biotic stress, but is also involved in the reaction of plants to abiotic stresses (Bragina et al. 2014) including high salinity and drought (Rolli et al. 2015; Yang et al. 2009). Recently, Castrillo et al. (2017) identified a genetic network controlling the phosphate stress response and influences the structure of the root microbiome community. Plant microbiota also have an influence on the composition of plant secondary metabolites (Schmidt al. 2014; Verginer et al. 2010). Peñuelas et al. (2014) showed that the removal of the floral microbiota of Sambucus nigra resulted in reduced floral terpene emission, which plays a key role in pollination and consequently in fruit and seed production. Recent studies also revealed the direct impact of the root microbiome on plant phenology (Panke-Buisse et al. 2014; Wagner et al. 2014). Additional essential roles of the plant microbiome for phenotypic and epigenetic plasticity as well as the evolution of plants were already suggested by Partida-Martínez and Heil (2011). The relevance of microorganisms for cold adaptation was shown for particular bacterial species, such as ice nucleation active bacteria that induce ice crystallization in leaves (Gurian-Sherman et al. 1993). In addition, plant-associated Pseudomonas and Burkholderia strains can induce chilling tolerance (Fernandez et al. 2012; Subramanian et al. 2015). However, the role of the whole plant microbiome in cold acclimation is still unexplored. The objective of this study was therefore to determine and compare the composition of the leafassociated bacteria of different Arabidopsis accessions that were previously classified as freezing tolerant or sensitive (Zuther et al. 2012) both before and after 14 days of cold acclimation at $4^{\circ} \mathrm{C}$.

\section{MATERIALS AND METHODS}

Plant material and experimental design. Samples of 10 previously characterized Arabidopsis accessions were used for the analysis of the bacterial communities. Four accessions with high freezing tolerance (tolerant) were selected (N14, Ms-0, N6, and Kn-0), five accessions with low freezing tolerance (sensitive) (Can-0, Alc-0, C24, Ita-0, and Ran-0), and the accession Col-0 with an intermediate freezing tolerance as the reference. The samples originated from a large study with 54 accessions performed in several separate experiments with three accessions each over a time period of 1 year described in Zuther et al. (2012) (Table 1). In this study, accessions were completely randomized with respect to their geographic origin. Plants were grown in a greenhouse with $16 \mathrm{~h}$ day,
$200 \mu \mathrm{E} \mathrm{m} \mathrm{m}^{-2} \mathrm{~s}^{-1}$ light intensity, and a temperature of $20 / 18^{\circ} \mathrm{C}$ day/night until 42 days after sowing (nonacclimated conditions, NA). For cold acclimation (ACC), plants were transferred to a growth chamber at $4^{\circ} \mathrm{C}, 16 \mathrm{~h}$ day length with $90 \mu \mathrm{Em}^{-2} \mathrm{~s}^{-1}$ at $60 \%$ humidity for an additional period of 14 days. Three biological replicates per accession and condition were collected with each replicate containing two fully developed rosette leaves each from a different plant.

Extraction of bacterial DNA. Samples were lyophilized and ground in a ball mill prior to isolation of total genomic DNA. DNA was extracted from $100 \mathrm{mg}$ of plant material using the FastDNA Spin Kit for Soil (MP Biomedicals, Santa Ana, CA) according to the manufacturer's protocol and stored at $-21^{\circ} \mathrm{C}$.

$16 S$ rRNA amplicon sample preparation, sequencing, and analysis. An amplicon sequencing using Illumina's MiSeq platform was applied to study the taxonomic structure of the bacterial communities using three biological replicates per studied accession and condition. 16S rRNA gene sequences containing the hypervariable region (V4) were amplified by the region-specific primer pair 515f and 806r (Caporaso et al. 2011) that carried Illumina cell flow adaptors and sample-specific barcodes. The polymerase chain reaction (PCR) involved specific PNA (peptide nucleic acid) oligos to preclude the amplification of plant-derived plastid DNA according to the adjusted protocol described by Lundberg et al. (2013). The PCR mixture $(30 \mu \mathrm{l})$ contained $1 \times$ Taq\&Go (MP Biomedicals, Illkirch, France), $0.2 \mu \mathrm{M}$ of each primer, $0.75 \mu \mathrm{M}$ of each mPNA and pPNA oligos, and $2 \mu \mathrm{l}$ of template DNA $\left(96^{\circ} \mathrm{C}\right.$ for $6 \mathrm{~min}, 30$ cycles at $96^{\circ} \mathrm{C}$ for $1 \mathrm{~min}, 78^{\circ} \mathrm{C}$ for $5 \mathrm{~s}, 54^{\circ} \mathrm{C}$ for $1 \mathrm{~min}$, $74^{\circ} \mathrm{C}$ for $1 \mathrm{~min}$, and final elongation at $74^{\circ} \mathrm{C}$ for $10 \mathrm{~min}$ ). Three independent PCR runs were performed for each sample and pooled prior to purification by applying the Wizard SV Gel and PCR CleanUp System (Promega, Madison, WI). After spectrophotometric estimation of DNA concentrations (Nanodrop 2000, Thermo Scientific, Wilmington, DE), equimolar aliquots of all samples were combined for amplicon sequencing using Illumina MiSeq chemistry v3 (250 bp paired-end) conducted by Eurofins Genomics (Ebersberg, Germany). Raw sequencing paired-end reads were assembled with default settings of PandaSeq software, version 2.8 (Masella et al. 2012). Low-quality reads defined as reads with an average quality score below 25, with more than one ambiguous base and a length $<250$ and $>260$ were removed using the Prinseq program, version 0.20.4 (Schmieder and Edwards 2011). In addition, barcodes and primers were trimmed by Prinseq software. Resulting sequence data sets are deposited at NCBI's Sequence Read Archive under the BioSample accession numbers SAMN07542821 to SAMN07542840.

TABLE 1

List of 10 accessions of Arabidopsis thaliana with information about their geographic origin, INRA ID, and freezing tolerance according to Zuther et al. (2012)

\begin{tabular}{lllcccc}
\hline Abb. & \multicolumn{1}{c}{ Name } & Origin & Latitude of origin & Longitude of origin & INRA accession ID & Category \\
\hline N6 & Karelia & RUS & 62.00 & 34.00 & Tolerant \\
\hline N14 & Sampo Mountain & RUS & 61.10 & 34.50 & TV & Tolerant \\
\hline Ms-0 & Moscow & RUS & 55.75 & 37.35 & AV & Tolerant \\
\hline Kn-0 & Kaunas & LIT & 54.90 & 23.54 & 70 AV & Tolerant \\
\hline Col-0 & Columbia & POL & 52.73 & 15.15 & Intermediate \\
\hline Can-0 & Canary Island & ESP & 28.00 & -15.30 & AV & Sensitive \\
\hline Ita-0 & Ibel Tazekka & MAR & 34.10 & -4.12 & AV & Sensitive \\
\hline C24 & & POR & 40.00 & -8.25 & NV \\
\hline Alc-0 & Alcala de Henares & ESP & 40.48 & -3.22 & Sensitive \\
\hline Ran & Randan & FRA & 40.50 & -2.00 & Sensitive \\
\hline
\end{tabular}


The processing of filtered reads to operational taxonomic units (OTUs) was done with the LotuS program using usearch at $97 \%$ similarity (Hildebrand et al. 2014). OTUs assigned to plant-derived chloroplasts and mitochondria were filtered out from the dataset by QIIME 1.9.0 (Caporaso et al. 2010). Since the applied primer pair 515f/806r (Caporaso et al. 2011) was reported to be biased toward archaeal 16S rRNA gene sequences, we decided to exclude those reads from the data set prior to further analysis. Bacterial 16S rRNA sequence data analysis and visualization were done by Phyloseq and Shinyphyloseq packages of R software (McMurdie and Holmes 2013, 2014; R Development Core Team 2011). To account for heterogeneity in read counts among samples, we employed the DESeq2 statistical package according to Love et al. (2014) to assess differential OTU abundances (Thorsen et al. 2016; Weiss et al. 2017). Statistical significance of differences regarding the relative taxonomic composition between tolerance categories was tested using analysis of variance (ANOVA). Alpha and beta diversity were analyzed using OTU table at an even read depth of 20,000. To test the significance of differences of alpha diversity metrics, we applied nonparametric $t$ test using Monte Carlo permutations (number of permutations: 999) and false discovery rate correction. Multidimensional scaling was performed based on a Bray-Curtis dissimilarity distance matrix. The statistical significance of groupings was tested by applying analysis of similarities (ANOSIM).

\section{RESULTS}

The structure of the bacterial community associated with Arabidopsis leaves changes during cold acclimation. The bacterial communities of the 10 Arabidopsis accessions that are either freezing tolerant (N6, N14, Ms-0, and Kn-0), intermediate (Col-0), or sensitive (Ran, Ita-0, Can-0, C24, Alc-0) according to Zuther et al. (2012), were highly diverse under both nonacclimated (NA) and cold acclimated (ACC) conditions. Overall, after removing sequences assigned to chloroplasts, mitochondria, and archaeal $16 \mathrm{~S}$ rRNA, 5,472 bacterial OTUs (16S rDNA at 97\% similarity cut-off) were identified in the final dataset comprising 1.479 million reads with a median of 20,110 reads per sample. After removing singleand doubleton OTUs, a total of 852 OTUs were detected in the 60 samples. The composition of bacterial communities at phylum level revealed Proteobacteria (40.7 to 56.8\%), Actinobacteria (19.7 to $27.5 \%$ ), Firmicutes (0.4 to 19.5\%), and Bacteroidetes (5.0 to $15.5 \%$ ), which dominated with more than $80 \%$ abundance in the three Arabidopsis freezing tolerance categories (Fig. 1A, Supplementary Table S1). At class level, Alpha-, Beta-, and Gammaproteobacteria (7 to $13.36 \%, 7$ to $38.86 \%, 9$ to $28.91 \%$ ), Actinobacteria (19.34 to $27.16 \%$ ), Bacilli (0.4 to $19.42 \%)$, Flavobacteria (2.06 to $7.73 \%$ ), Sphingobacteria (1.87 to $5.57 \%$ ), and Saprospirae (0.9 to $4.21 \%$ ) were the major representative taxa. Noticeably, the genus Buchnera, known as an obligate intracellular symbiont of aphid species, represents an average of $4.7 \%$ of the bacterial communities. The assignment to Buchnera, was confirmed by stringent alignment of all reads contributing to the respective OTUs. All reads show high nucleotide similarities (99\%) with 16S rRNA gene reference sequences of Buchnera. Although most of the bacterial groups were found among all tolerance categories under both growth conditions, some classes such as TK17 and Ellin6529 from the Chloroflexi phylum were only present after cold acclimation and TM7-3 from TM7 was only present in the sensitive category (Fig. 1B). No statistically significant differences of relative abundances were observed for the dominant taxa at phylum and class level between acclimated and nonacclimated plants within accession $(P \leq 0.05)$. Hence, to detect the impact of cold conditions on dominant members within the bacterial community, we studied the 100 most abundant among the
852 identified OTUs in depth. They were assigned to nine classes of Alpha-, Beta-, Gammaproteobacteria, Actinobacteria, Bacilli, Flavobacteriia, Saprospirae, Sphingobacteriia, and Verrucomicrobiae. The distribution and composition of these top 100 OTUs varied among accessions (Fig. 2). In general, OTUs belonging to Gammaproteobacteria and Bacilli in tolerant and Bacilli and Actinobacteria in sensitive accessions and to Betaproteobacteria and Actinobacteria in Col-0 were found to dominate the respective bacterial communities (Fig. 2). Moreover, the relative abundance of particular OTUs within the microbial communities in all tolerance categories was altered during cold acclimation, e.g., the abundance of OTUs assigned to Gammaproteobacteria was higher in the tolerant category under cold acclimated than under nonacclimated conditions (Fig. 2).

Cold acclimation was accompanied by a shift of bacterial diversity. The number of observed OTUs at a depth of 20,000 reads varied from 243 to 718 in the 60 samples (Supplementary Fig. S1). In the course of cold acclimation, the number of observed OTUs decreased within respective tolerance categories; however, a statistically significant decrease (740 to 530 bacterial species) was only detected between nonacclimated and cold acclimated plants of the accession Col-0 (Fig. 3A). In addition, the evenness indicated by the Shannon index was not significantly different between tolerance categories or within tolerance categories and both conditions except for a decrease in cold acclimated Col-0 compared with nonacclimated plants of the same accession (5.6 to 3.9) (Fig. 3B). The richness and evenness of bacterial species among the individual accessions showed a wide variation (Supplementary Fig. S2). The bacterial communities of the accessions N6 and N14 (tolerant) and Col-0 (intermediate) had the highest richness and Alc-0 (sensitive), Col-0 (intermediate), and Ms-0 (tolerant) had the highest Shannon index under nonacclimated condition compared with all other accessions. Under acclimated conditions, the highest bacterial richness was found in N6 (tolerant), Alc-0 (sensitive), N14 (tolerant), and RAN (sensitive) and the highest evenness in N6 (tolerant), C24, and Alc-0 (sensitive).

Multidimensional scaling with the proportional normalized OTU tables and the distance matrix calculated using Bray-Curtis showed a clear separation into two groups under nonacclimated conditions (ANOSIM, $P=0.05$ ). One group contains three sensitive (Alc-0, $\mathrm{C} 24$, and Ran) and the other group contained all tolerant accessions and two sensitive accessions (Ita-0 and Can-0) together with Col-0 (Fig. 4A). Except for the accessions N6 (tolerant) and Ita-0 (sensitive), cold acclimation caused a statistically significant shift of bacterial communities within each Arabidopsis accessions (Supplementary Table S2, $P \leq 0.05$ ) and the differentiation of the bacterial community structure of the different accessions are even more distinct (Fig. 4B). The results revealed a separation into a tolerant group (N6, N14, Ms-0, and Kn-0), a sensitive group I (C24, Alc-0, and Ran) and a sensitive group II (Ita-0 and Can- 0 ) which also contains Col-0 (ANOSIM, $P=0.002$ ). The stronger separation of the bacterial community structure under cold acclimated than under nonacclimated conditions is also visualized by interactive network graphics ( 0.32 as Bray-Curtis distance threshold) based on the bacterial community of each accession (Supplementary Fig. S3).

Cold temperature affected the bacterial core. Since core species are critical to the function of a microbial community, we identified bacterial OTUs representing the core in the leaves of each $A$. thaliana accession under both conditions. For that, the OTUs present in at least $80 \%$ of samples in each tolerance category were chosen as a representation of the bacterial core (Supplementary Table S3). By visualizing the shared OTUs between freezing tolerance categories under both growth conditions, Venn diagrams clearly showed the increase of specific core bacterial species in the sensitive group (I) and the tolerant group during cold acclimation compared with non- acclimated samples (Fig. 5A and B). In contrast, this was not the case for the 
A

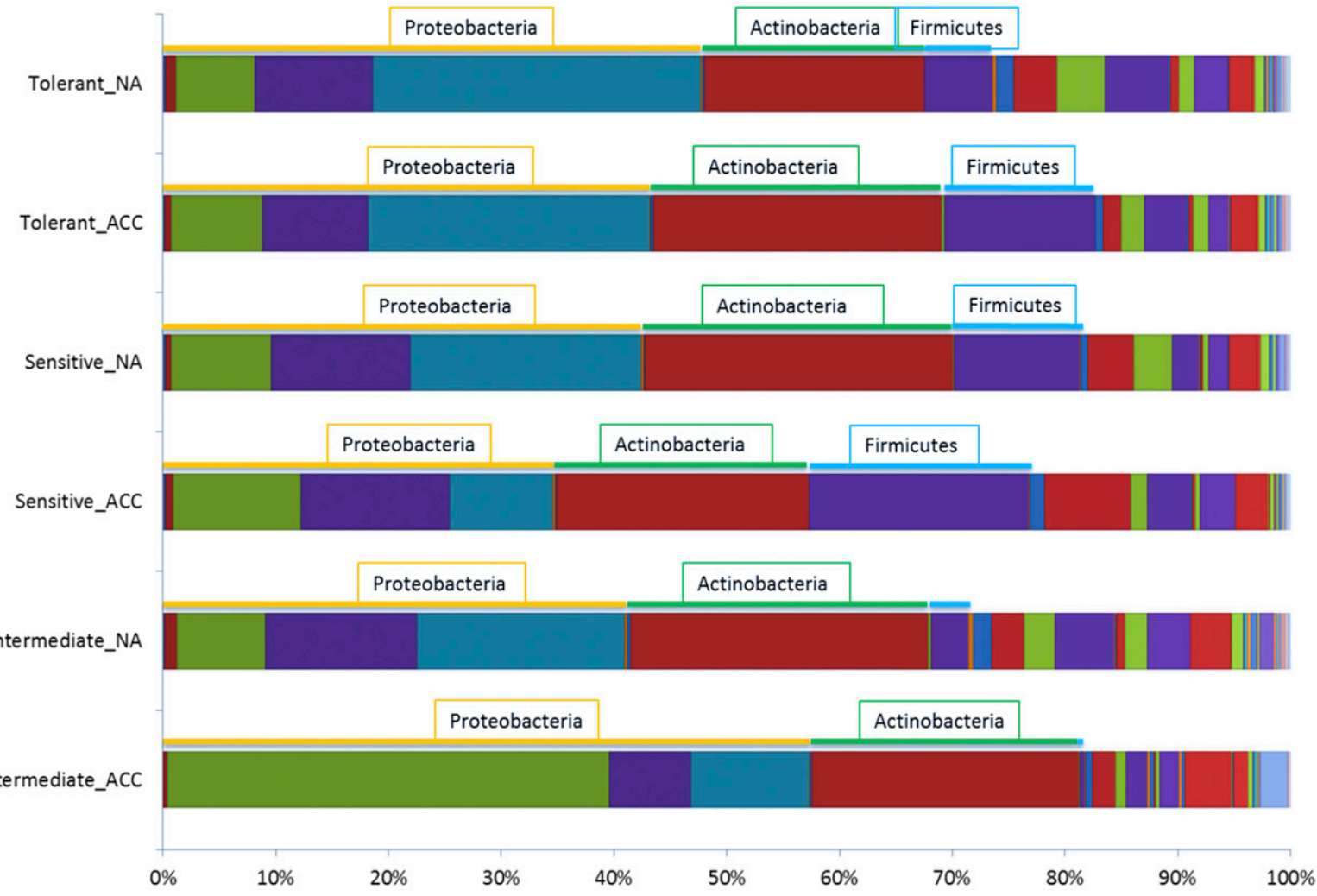

B

\begin{tabular}{|c|c|c|c|c|c|c|c|c|c|c|c|c|c|c|c|c|c|c|c|c|}
\hline & & & & & & & & & & & & & 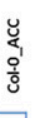 & & 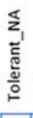 & & 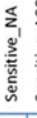 & & & \\
\hline roteobacteria Unknown & * & * & * & * & * & * & - Proteobacteria Deltaproteobacteria & * & * & * & * & * & * & I Proteobacteria Betaproteobacteria & * & * & * & * & * & * \\
\hline roteobacteria Alphaproteobacteria & * & $*$ & * & * & $*$ & * & - Proteobacteria Gammaproteobacteria & * & * & * & * & * & * & Actinobacteria Acidimicrobiia & * & * & * & * & * & * \\
\hline Actinobacteria Thermoleophilia & $*$ & * & $*$ & $*$ & $*$ & * & Actinobacteria Actinobacteria & * & $*$ & $*$ & * & * & * & Firmicutes Clostridia & * & * & * & * & * & * \\
\hline Firmicutes Bacilli & * & * & * & $*$ & $*$ & * & Eacteroidetes SM1A07 & & & & & * & & Bacteroidetes VC2_1_Bac22 & * & $*$ & * & $*$ & $*$ & * \\
\hline Bacteroidetes Cytophagia & * & $*$ & $*$ & * & $*$ & * & Bacteroidetes Flavobacteriia & * & * & $*$ & $*$ & $*$ & * & Bacteroidetes Saprospirae & $*$ & * & $*$ & $*$ & $*$ & * \\
\hline Sacteroidetes Sphingobacteriia & * & * & $*$ & * & * & * & = Bacteroidetes Unknown & $*$ & & * & $*$ & $*$ & & E Verrucomicrobia Pedosphaerae & $*$ & * & $*$ & * & $*$ & * \\
\hline /errucomicrobia Methylacidiphilae & $*$ & * & $*$ & & * & * & -Verrucomicrobia Opitutae & $*$ & $*$ & $*$ & * & * & * & Verrucomicrobia Spartobacteria & * & * & * & $*$ & $*$ & * \\
\hline Verrucomicrobia Verrucomicrobiae & * & * & $*$ & * & * & * & $\varpi$ Planctomycetes BD7-11 & & * & & & * & $*$ & N Planctomycetes OM190 & & $*$ & * & & & $*$ \\
\hline Planctomycetes Phycisphaerae & * & $*$ & $*$ & $*$ & * & $*$ & EPlanctomycetes Planctomycetia & * & * & $*$ & * & $*$ & * & =Chloroflexi Ellin6529 & & * & & * & & * \\
\hline Chloroflexi Ktedonobacteria & & & & & $*$ & & =Chloroflexi Chloroflexi & & & $*$ & & $*$ & & $=$ Chloroflexi TK10 & & $*$ & & & * & \\
\hline Chloroflexi TK17 & & * & & $*$ & & $*$ & EChloroflexi Anaerolineae & ${ }^{*}$ & * & * & * & * & * & E Chloroflexi Thermomicrobia & $*$ & * & $*$ & * & $*$ & * \\
\hline Acidobacteria Acidobacteria-6 & $*$ & * & * & * & * & * & 플 Acidobacteria Acidobacteriia & * & $*$ & * & * & * & * & $\square$ Acidobacteria Solibacteres & * & $*$ & $*$ & * & $*$ & $*$ \\
\hline Acidobacteria Chloracidobacteria & ${ }^{*}$ & $*$ & $*$ & * & $*$ & $*$ & acidobacteria DA052 & * & * & $*$ & $*$ & * & $*$ & Ehlamydiae Chlamydiia & * & * & * & * & $*$ & $*$ \\
\hline TM6 SJA-4 & * & ${ }^{*}$ & $*$ & * & $*$ & * & Eusobacteria Fusobacteriia & & & $\begin{array}{ll}* \\
*\end{array}$ & & & * & Gemmatimonadetes Gemm-1 & * & * & $*$ & $*$ & * & \\
\hline $\begin{array}{l}\text { Gemmatimonadetes Gemmatimonadetes } \\
\text { OD1 ZB2 }\end{array}$ & $\begin{array}{l}* \\
*\end{array}$ & * & $\begin{array}{ll}* \\
*\end{array}$ & $\begin{array}{ll}* \\
*\end{array}$ & * & $\begin{array}{ll}* \\
*\end{array}$ & $\begin{array}{l}\text { Eemmatimonadetes Gemm-3 } \\
=\text { Cyanobacteria } 4 \text { COd-2 }\end{array}$ & * & * & $\begin{array}{l}* \\
*\end{array}$ & $\begin{array}{l}* \\
*\end{array}$ & * & * & $=$ NKB19 TSBW08 & * & & & & * & \\
\hline Cyanobacteria Synechococcophycideae & * & * & * & * & $*$ & * & ECyanobacteria Unknown & * & * & * & * & * & * & = Cyanobacteria ML635J-21 & * & * & * & * & $*$ & $*$ \\
\hline FBP Unknown & * & $*$ & $*$ & * & * & $*$ & $\equiv$ Fibrobacteres Fibrobacteria & * & $*$ & * & * & * & * & \# Cyanobacteria Oscillatoriophycide & * & & * & * & & \\
\hline Armatimonadetes Fimbriimonadia & * & $*$ & * & * & * & * & $\approx$ Armatimonadetes 0319.6E2 & * & * & * & * & $*$ & * & $\|$ Armatimonadetes Armatimonadia & * & * & * & * & * & \\
\hline BRC1 PRR-11 & * & * & * & * & * & * & EChlorobi Unknown & * & * & & * & $*$ & & BHI80-139 Unknown & * & & & & & \\
\hline SAR406 AB16 & * & * & $*$ & * & * & $*$ & $=$ SBR1093 VHS-B5-50 & & * & & & & * & $=$ Chlorobi OPB56 & $*$ & $*$ & $*$ & * & $*$ & $*$ \\
\hline Spirochaetes Spirochaetes & $*$ & & & & * & & Tenericutes Mollicutes & * & & * & & & & Spirochaetes Leptospirae & & $*$ & $*$ & & & $*$ \\
\hline Thermi Deinococci & & * & * & * & & * & TM7 TM7-1 & * & * & * & * & * & * & Tenericutes Unknown & * & * & $*$ & & & \\
\hline TM7 TM7-3 & & & * & * & & & WPS-2 Unknown & * & & * & * & $*$ & & $=$ TM7 SC3 & * & * & & & * & \\
\hline
\end{tabular}

Fig. 1. Taxonomic composition of bacterial communities in the phyllosphere of different Arabidopsis accessions grown under nonacclimated (NA) or cold acclimated (ACC) conditions. A, Relative composition at phylum level analyzed by Illumina MiSEquation $16 S$ rRNA gene amplicon sequencing. The color code of bacterial classes can be found in B. Ten Arabidopsis accessions were grouped according to their freezing tolerance into a tolerant (N14, Ms-0, N6, and Kn-0) and a sensitive (Can-0, Alc-0, C24, Ita-0, and Ran-0) group, and Col-0 is shown as reference. No statistically significant differences of relative abundances were observed for the dominant taxa at phylum and class level between acclimated and nonacclimated plants within accession $(P \leq$ 0.05). B, Microbial classes detected in the three differently tolerant categories. The exclusive presence of the respective microbial class within a freezing tolerance group is indicated by asterisks. 
sensitive group (II) and Col-0, where a significant reduction of bacterial richness in response to cold was detected.

Common responses of bacterial communities to cold. We performed an additional statistical analysis (Love et al. 2014; McMurdie and Holmes 2014) between the sensitive and tolerant categories under nonacclimated and cold acclimated conditions to investigate whether there were common microbial responses among the sensitive and tolerant accessions during cold acclimation. Among the 852 OTUs, 53 and 46 OTUs were significantly different $(P<0.05)$ after cold acclimation compared with nonacclimated

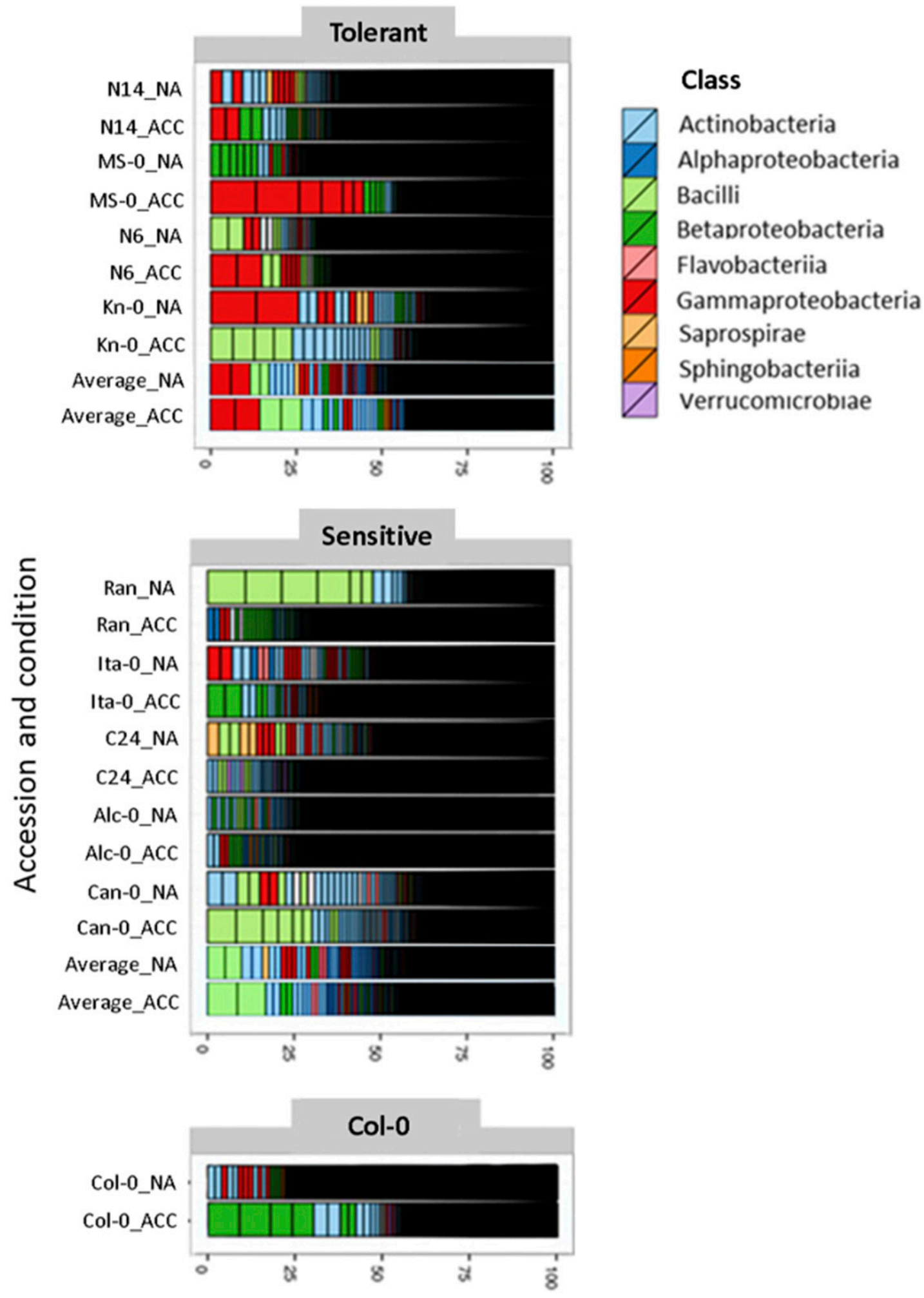

Fig. 2. Percentage of sequences representing the 100 most abundant operational taxonomic units (OTUs) in the leaves of different Arabidopsis accessions under nonacclimated (NA) or cold acclimated (ACC) conditions. The distribution of the 100 most abundant OTUs among 852 identified OTUs in the dataset is shown for each accession and condition within the respective tolerance category and additionally averaged for the tolerant and sensitive category for both conditions separately. 
samples in the sensitive and tolerant category, respectively (Supplementary Table S4). Among these, eight OTUs overlapped between the tolerant and sensitive categories. The OTU_2839 (genus Truepera), OTU_2099 and OTU_2390 (Nevskia), OTU_2453, and OTU_5856 (genus unknown, phylum Chloroflexi) showed significantly $(P<0.05)$ higher abundance under cold acclimated compared with nonacclimated conditions in both sensitive and tolerant accessions, while OTU_779 (Sphingopyxis), OTU_1903 (Luteibacter), and OTU_1237 (genus: unknown, phylum: Bacteroidetes) showed significantly $(P<0.05)$ lower abundance (Fig. 6). This indicates that part of the microbial community of $A$. thaliana leaves responded similarly to cold in all investigated genotypes.

The main bacterial responders in tolerant Arabidopsis accessions. To identify the OTUs separating the tolerant from the sensitive accessions, we performed a pairwise statistical analysis between the tolerant and the sensitive category. In comparisons between the tolerant versus sensitive group (I) and sensitive group (II) and Col-0, 261, 224, and 122 OTUs, respectively, were significantly different $(P<0.05)$ (Supplementary Table S5). Among these different OTUs, 29 were shared between all three comparisons. These common OTUs separate the tolerant category significantly from all others under cold acclimated conditions (Table 2). To visualize, whether these 29 OTUs separated the tolerant from the sensitive accessions and Col-0, we performed a nonmetric multidimensional scaling plot with the replicate average for each accession (Supplementary Fig. S4). A separation of tolerance categories by these OTUs could be verified. In the next step, we used ANOVA to analyze whether these 29 OTUs were distributed equally among the tolerant accessions (Supplementary Table S6). OTU_1795, OTU_2099, OTU_2390, OTU_270, OTU_2769, OTU_3360, OTU_4007, OTU_4288, OTU_4622, OTU_4915, OTU_67, and OTU_878 were significantly $(P<0.05)$ different
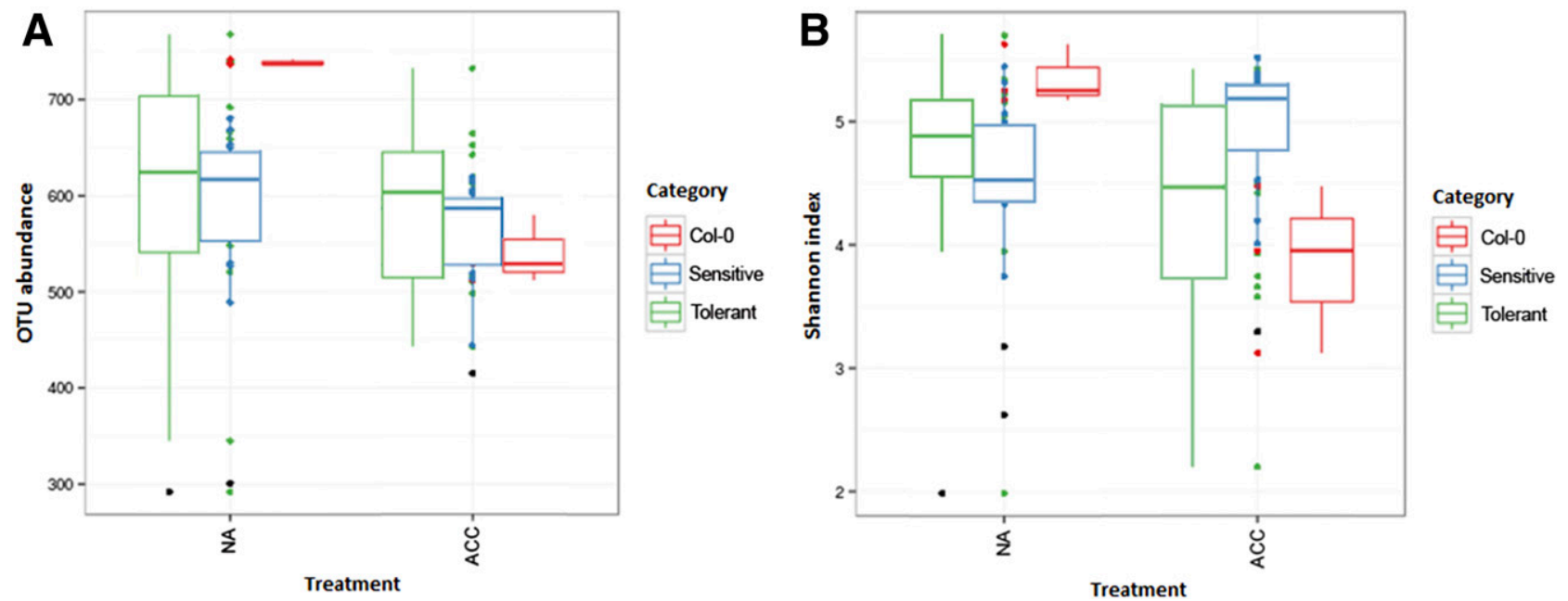

Fig. 3. Alpha diversity of bacterial communities associated with Arabidopsis accessions of different tolerance categories under nonacclimated (NA) or cold acclimated (ACC) conditions. A, Number of observed operational taxonomic units (OTUs) at $97 \%$ similarity and even depth of 20,000 reads for Arabidopsis accessions grouped in tolerance categories (Col-0, $n=3$ per condition; sensitive, $n=15$ per condition; and tolerant, $n=12$ per condition). Comparing nonacclimated and cold acclimated plants of the same tolerance category, a nonsignificant decrease in the number of observed OTUs after cold acclimation was observed for all categories, except for Col-0, which significantly decreased. B, The Shannon diversity index was calculated based on the distribution of values across all tolerance categories. Error bars ( $\mathbf{A}$ and $\mathbf{B})$ represent the standard deviation (Col-0, $n=3$ per condition; sensitive, $n=15$ per condition; and tolerant, $n=12$ per condition). Except for Col-0, no statistically significant differences between nonacclimated and cold acclimated plants of the same tolerance category were observed.
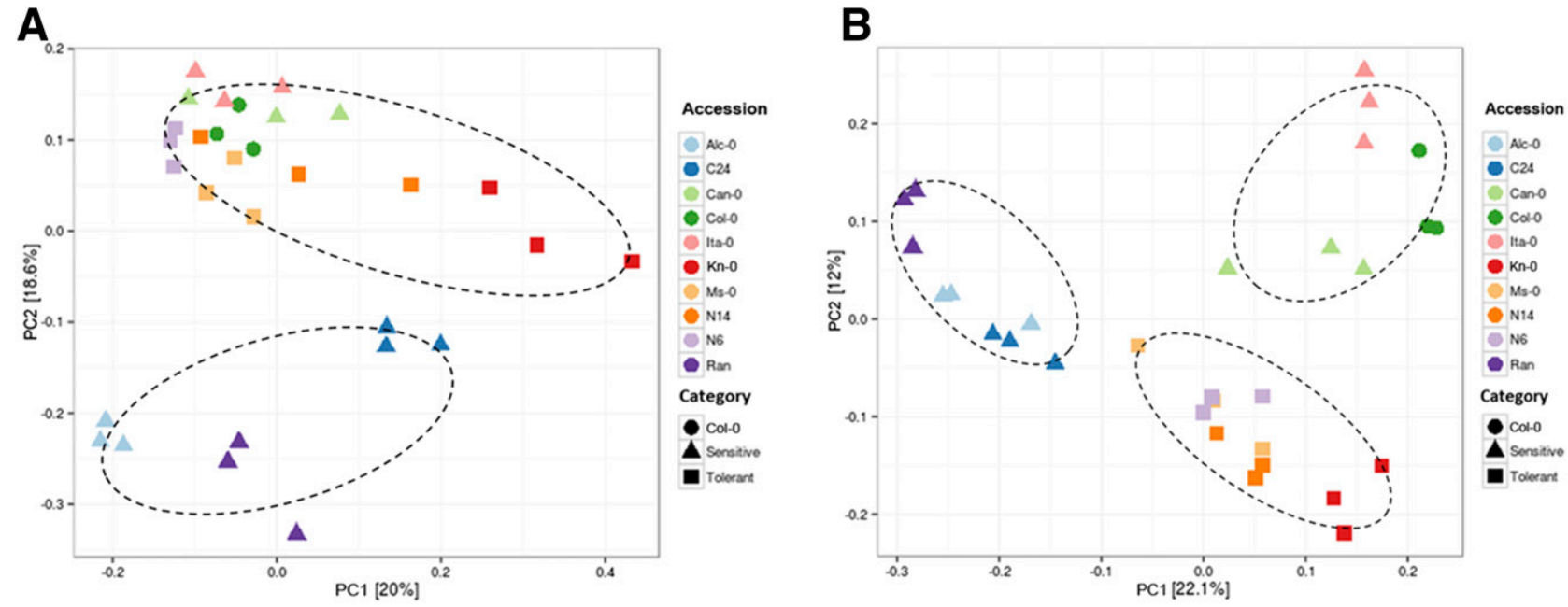

Fig. 4. Bray-Curtis-based multidimensional scaling of bacterial communities under $\mathbf{A}$, nonacclimated (NA) and $\mathbf{B}$, cold acclimated (ACC) conditions. 
between tolerant accessions (Table 2). Some of them were part of core bacterial species such as OTU_2099, OTU_2390, OTU_270, OUT_4007, OTU_4622, OTU_4915, and OTU_67, suggesting accession-specific responses of bacterial communities to cold (Table 2). The abundance of these 29 OTUs in the different accessions was visualized in a heatmap (Fig. 7). Interestingly OTU_4869 was only present in tolerant accessions and Col-0, while OTU_4070 was only detected in tolerant accessions after cold acclimation. In contrast, OTU_2206 was only present in sensitive accessions and Col-0.
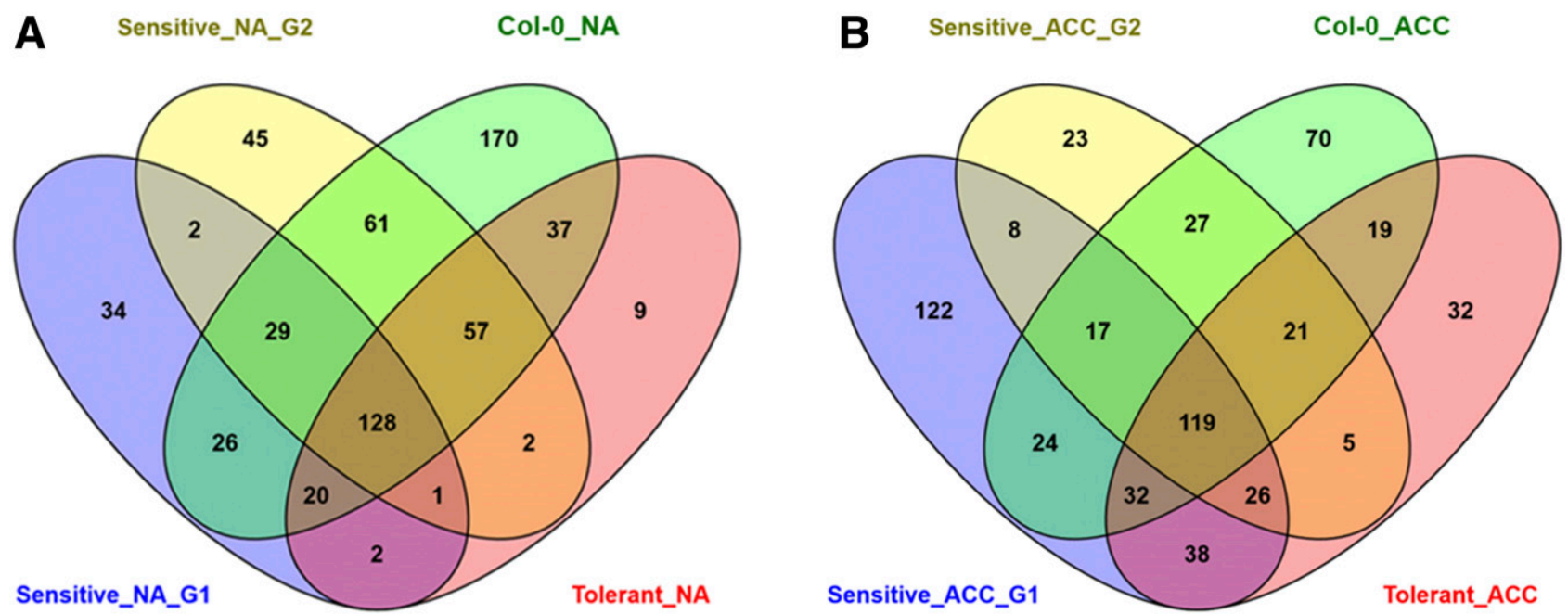

Fig. 5. Venn diagrams of the core bacterial operational taxonomic units (OTUs) of Arabidopsis accessions from three different tolerance categories. Each number refers to an OTU that constitute the core of different tolerance categories classified under A, nonacclimated (NA) or B, cold acclimated (ACC) conditions. The sensitive tolerance category was split into two groups, group I and group II, according to the network analysis.

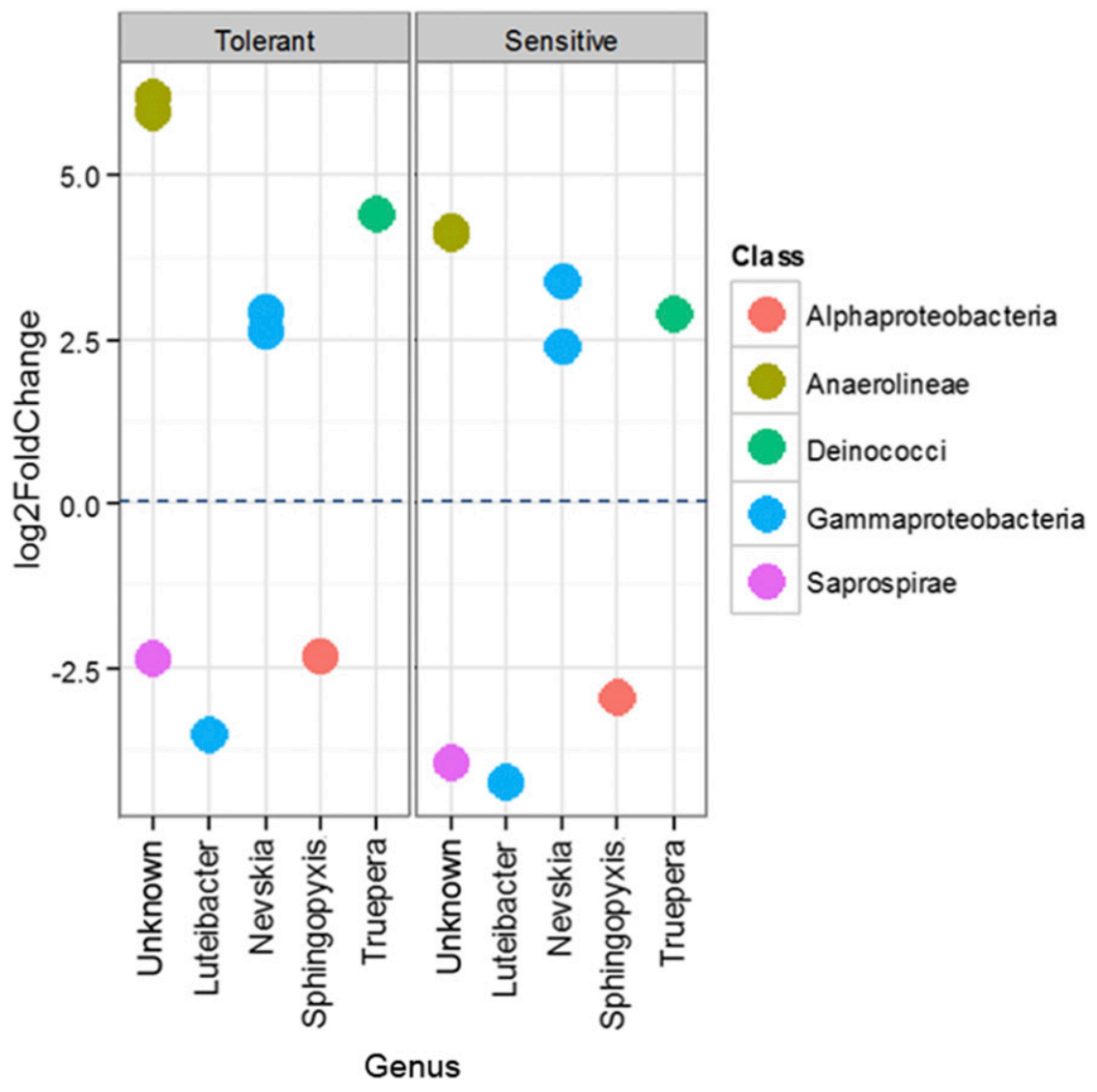

Fig. 6. Fold change (log2) of common operational taxonomic units (OTUs) in bacterial communities of Arabidopsis accessions of the sensitive and tolerant categories between nonacclimated and cold acclimated conditions. The unknown genera are either from the phylum Chloroflexi and represented by OTU_2453 and OTU_5856 or from the phylum Bacteroidetes and represented by OTU_1237. Luteibacter is represented by OTU_1903, Nevskia by OTU_2099 and OTU_2390, Sphingopyxis by OTU_779, and Truepera by OTU_2839. 


\section{DISCUSSION}

The natural genetic variation among Arabidopsis accessions results in different levels of tolerance under various environmental conditions (Weigel 2012). Here, we show that the response of bacterial community composition to cold acclimation significantly correlates with freezing tolerance levels of different Arabidopsis accessions, which has previously been related to differences in gene expression and metabolite content (Hincha et al. 2012).

In all Arabidopsis accessions, a significant change of bacterial community structure and diversity was observed following the cold acclimation period at $4^{\circ} \mathrm{C}$ for 14 days. Our study included bacteria associated with leaves comprising the phyllosphere as well as the endosphere. While it has been reported that the plant phyllosphere is a dynamic environment subjected to large variation in temperature, moisture, and radiation throughout the day and night (Bai et al. 2015; Turner et al. 2013; Vorholt 2012), the endosphere harbors a much more stable microbial community (Hardoim et al. 2015). Even though in Arabidopsis the species compositions in both microenvironments were reported to be similar (Bodenhausen et al.
2013). Under nonacclimated conditions, the composition of bacterial communities associated with leaves showed a genotypespecific signature as already reported for Arabidopsis (Bai et al. 2015) and for several crop species (Berg and Smalla 2009; Bulgarelli et al. 2015; Cardinale et al. 2014). All these studies describe the impact of diversification and especially breeding on the microbiome composition but the functional relevance remains largely unexplored. Interestingly, in our study, the genotype specificity of bacterial communities after cold acclimation was even more distinct. This confirms our hypothesis that the bacterial communities associated with Arabidopsis leaves are influenced by cold acclimation and differs between freezing tolerant and sensitive accessions. Moreover, the identified relationship between the level of freezing tolerance and the bacterial responses to cold acclimation represents a first functional indication. In addition to the changed structure in response to the cold, we observed the tendency that bacterial diversity decreased. Bacterial diversity is a crucial factor for ecosystem function and plant health (Berg et al. 2017; Laforest-Lapointe et al. 2017). However, in general, all samples showed an unexpected high diversity and 852 OTUs were identified.

TABLE 2

Twenty-nine selected operational taxonomic units (OTUs) that separate tolerant Arabidopsis accessions from the sensitive accessions or Col-0 under cold acclimated conditions ${ }^{a}$

\begin{tabular}{|c|c|c|c|c|c|c|c|c|c|}
\hline \multirow[b]{2}{*}{ OTU } & \multicolumn{7}{|c|}{ Taxonomy } & \multirow{2}{*}{$\begin{array}{l}\text { Analysis } \\
\text { of variance }\end{array}$} & \multirow{2}{*}{$\begin{array}{l}\text { Core } \\
\text { microbiome } \\
\text { (tolerant) }\end{array}$} \\
\hline & Kingdom & Phylum & Class & Order & Family & Genus & Species & & \\
\hline OTU_1016 & Bacteria & Acidobacteria & Chloracidobacteria & $R B 41$ & Ellin6075 & $?$ & $?$ & & C \\
\hline OTU_1406 & Bacteria & Proteobacteria & Alohaproteobacteria & Caulobacterales & Caulobacteraceae & Arthrospira & fusiformis & & \\
\hline OTU_1539 & Bacteria & Proteobacteria & Alphaproteobacteria & Rhizobiales & Hyphomicrobiaceae & Devosia & $?$ & & $\mathrm{C}$ \\
\hline OTU_164 & Bacteria & Bacteroidetes & Flavobacteria & Flavobacteriales & Flavobacteriaceae & Flavobacterium & $?$ & & $\mathrm{C}$ \\
\hline OTU_1745 & Bacteria & Verrucomicrobia & Spartobacteria & Chthoniobacterales & Chthoniobacteraceae & heteroC45_4W & $?$ & & $\mathrm{C}$ \\
\hline OTU_1795 & Bacteria & Bacteroidetes & Sphingobacteriia & Sphingobacteriales & $?$ & $?$ & $?$ & * & \\
\hline OTU_1951 & Bacteria & Actinobacteria & Actinobacteria & Actlnomycetales & Promicromonosporaceae & Promicromonospora & $?$ & & C \\
\hline OTU_1968 & Bacteria & Actinobacteria & Actinobacteria & Actinomycetales & Nocardioidaceae & Nocardioides & plantarum & & \\
\hline OTU_2099 & Bacteria & Proteobacteria & Gammaproteobacteria & Xanthomonadales & Sinobacteraceae & Nevskia & ramosa & * & C \\
\hline OTU_2206 & Bacteria & Bacteroidetes & Sphingobacteriia & Sphingobacteriales & Sphingobacteriaceae & $?$ & $?$ & & \\
\hline OTU_2390 & Bacteria & Proteobacteria & Gammaproteobacteria & Xanthomonadales & Sinobacteraceae & Nevskia & ramosa & * & $\mathrm{C}$ \\
\hline OTU_270 & Bacteria & Proteobacteria & Gammaproteobacteria & Enterobacteriales & Enterobacteriaceae & Buchnera & $?$ & * & $\mathrm{C}$ \\
\hline OTU_2769 & Bacteria & Verrucomicrobia & Methylacidiphilae & Methylacidiphilales & LD19 & $?$ & $?$ & * & \\
\hline OTU_3072 & Bacteria & Bacteroidetes & Sphingobacteriia & Sphingobacteriales & Sphingobacteriaceae & $?$ & $?$ & & \\
\hline OTU_3360 & Bacteria & Bacteroidetes & Sphingobacteriia & Sphingobacteriales & $?$ & $?$ & $?$ & * & \\
\hline OTU_3470 & Bacteria & Proteobacteria & Alphaproteobacteria & Rhodospirillales & Rhodospirillaceae & Phaeospirillum & fulvum & & $\mathrm{C}$ \\
\hline OTU_4007 & Bacteria & Proteobacteria & Gammaproteobacteria & Xanthomonadales & Sinobacteraceae & $?$ & $?$ & * & C \\
\hline OTU_4070 & Bacteria & Proteobacteria & Alphaproteobacteria & Sphingomonadales & Sphingomonadaceae & Sphingomonas & wittichii & & $\mathrm{C}$ \\
\hline OTU_4177 & Bacteria & TM6 & SJA-4 & $?$ & $?$ & $?$ & $?$ & & \\
\hline OTU_4183 & Bacteria & Bacteroidetes & Flavobacteriia & Flavobacteriales & Flavobacteriaceae & Flavobacterium & $?$ & & \\
\hline OTU_4223 & Bacteria & Actinobacteria & Actinobacteria & Actinomycetales & Microbacteriaceae & Rathayibacter & caricis & & $\mathrm{C}$ \\
\hline OTU_4288 & Bacteria & TM6 & SJA-4 & $?$ & $?$ & $?$ & $?$ & * & \\
\hline OTU_4622 & Bacteria & Proteobacteria & Gammaproteobacteria & Xanthomonadales & Sinobacteraceae & $?$ & $?$ & * & $\mathrm{C}$ \\
\hline OTU_4869 & Bacteria & Verrucomicrobia & Methylacidiphilae & Methylacidiphilales & $L D 19$ & $?$ & $?$ & & \\
\hline OTU_4915 & Bacteria & Proteobacteria & Alphaproteobacteria & Rhizobiales & Bradyrhizobiaceae & Bosea & genosp. & * & $\mathrm{C}$ \\
\hline OTU_5871 & Bacteria & Proteobacteria & Alphaproteobacteria & Rhodospirillales & Rhodospirillaceae & Phaeospirillum & fulvum & & $\mathrm{C}$ \\
\hline OTU_67 & Bacteria & Proteobacteria & Gammaproteobacteria & Enterobacteriales & Enterobacteriaceae & Buchnera & $?$ & * & $\mathrm{C}$ \\
\hline OTU_878 & Bacteria & Actinobacteria & Actinobacteria & Actinomycetales & Nocardioidaceae & Nocardioides & plantarum & * & \\
\hline OTU_991 & Bacteria & Proteobacteria & Alphaproteobacteria & Rhizobiales & Hyphomicrobiaceae & Devosia & $?$ & & C \\
\hline
\end{tabular}

a The abundance of these OTUs was significantly $(P<0.05)$ different in Arabidopsis accessions of the tolerant category compared with the sensitive category or Col-0 under cold acclimated conditions. An analysis of variance indicated a significantly different abundance of several OTUs within tolerant accessions (shown by asterisk, $P<0.05$ ). Seventeen shared OTUs (more than $80 \%$ abundance in each category), representing the core microbiome within tolerant accessions, are indicated by $\mathrm{C}$. 
Comparing sensitive and tolerant Arabidopsis accessions, we found category-specific differences. Some members of the bacterial community such as OTU_270 and OTU_67 (Buchnera spp.) were more abundant in tolerant than in sensitive accessions. Moreover, their abundance increased in tolerant accessions after cold acclimation. Although Buchnera spp. are known as obligate intracellular symbiont of aphid species (Wilson and Duncan 2015), members of the genus were frequently detected in plant microbiomes (Ottesen et al. 2013). The occurrence of these species could, however, also originate from aphids associated with leaves. In contrast, OTU_3072 and OTU_2206 (Sphingobacteriaceae) were mostly present in sensitive accessions and not in the tolerant group. These discriminating bacterial species could potentially contribute to temperature adaptation and affect the tolerance/sensitivity of Arabidopsis accessions to freezing. However, this hypothesis must be tested experimentally by isolation and application of single bacterial strains.

Cold acclimation affected the relative composition of leafassociated bacterial communities in all Arabidopsis genotypes. We identified eight common OTUs responding to the adaptation process in both freezing tolerance categories. Five OTUs comprising the genera Nevskia, Truepera, and an unknown genus within the phylum Chloroflexi were observed to be enriched in cold acclimated plants. In contrast, three OTUs assigned to Luteibacter, Sphingopyxis, and an unknown genus within the phylum Bacteroidetes appears to be depleted. Although all responding taxa were frequently ascribed to plant microbiota, their specific role in the association with plants subjected to abiotic stresses is unclear. In rice plants, the relative abundance of several OTUs, including OTUs assigned to Chloroflexi, increased in response to drought stress (Santos-Medellín et al. 2017). The enrichment of particular bacteria was assumed to be mediated by the accumulation of compatible solutes, a similar protective mechanism that is induced by cold stress in Arabidopsis (Smallwood and Bowles 2002). A major determinant of epiphytic colonization is the availability of carbon-containing nutrients (Lindow and Brandl 2003). Sugars can be washed off from the surface of leaves (Mercier and Lindow 2000), but sugar distribution over the leaf area is heterogenous, probably due to localized leakage or transport from underlying cells (Leveau and Lindow 2001). Studies on bean leaves with bacterial biosensors consisting of Erwinia herbicola harboring a sucrose and fructose-responsive $s c r Y$ promoter fused to a $g f p$ or inaZ (ice nucleating protein) reporter gene, showed GFP fluorescence and ice nucleation in a sugar-dependent manner (Miller et al. 2001). During cold acclimation, the content of compatible solutes such as sugars

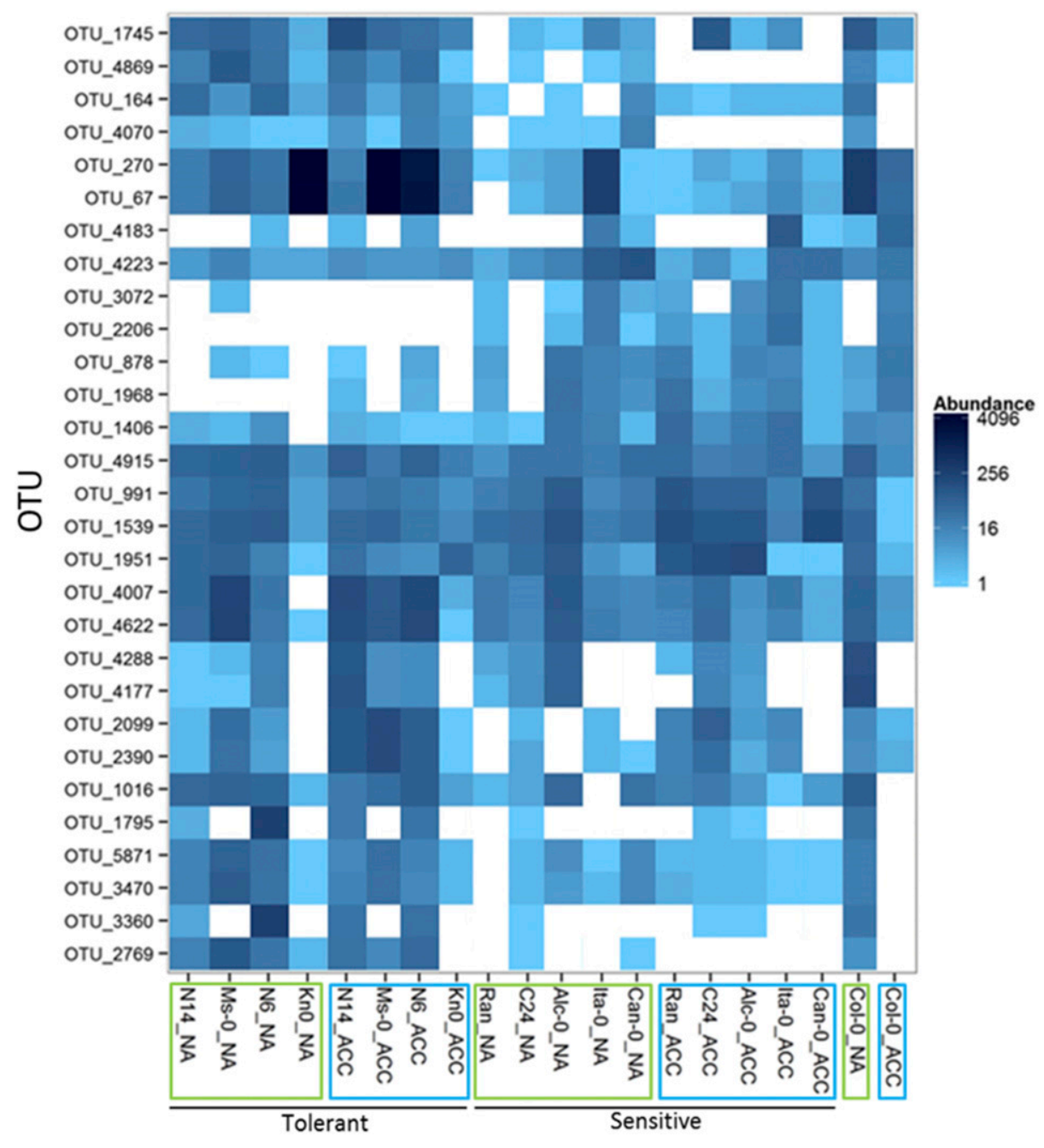

Fig. 7. Heat map depicting the relative bacterial diversity abundance in tolerant and sensitive accessions and Col-0 under nonacclimated (NA) or cold acclimated (ACC) conditions. The colored lines around the accessions indicate the treatments (green = nonacclimated [NA], blue = cold acclimated $[A C C]$ ). The colors in the heat map indicate the abundance of each operational taxonomic unit (OTU) within the samples. An absence of the specific bacterial community is shown in white. All presented OTUs are referred to in Table 2. 
and amino acids is strongly increased in Arabidopsis leaves where the content of glucose, fructose, sucrose, and raffinose is strongly correlated with freezing tolerance across diverse accessions (Korn et al. 2008; Rohde et al. 2004; Zuther et al. 2012). Investigating 54 Arabidopsis accessions, we showed that sensitive accessions accumulated lower amounts of sugars in rosette leaves after cold acclimation compared with tolerant accessions in rosette leaves (Zuther et al. 2012). Whether this difference contributes to the different distribution of OTUs in the bacterial community of differing tolerant accessions is not known and has to be investigated in the future. To our knowledge there is currently no information available on how leaf sugar content and composition affect the bacterial leaf microbiome at all. However, protection of bacteria during freezing involves the accumulation of compatible solutes such as sugars, polyols, amino acids, polyamines (Margesin 2011), and raffinose, which is highly correlated with freezing tolerance in Arabidopsis accessions, was found in Pseudomonads isolated from the rhizosphere of Himalayan plants grown at $4^{\circ} \mathrm{C}$ (Bisht et al. 2013). Burkholderia phytofirmans PsJN, a plant growth-promoting rhizobacterium, improved the tolerance of grapevine to cold by modulating plant carbohydrate metabolism in inoculated nonacclimated plants (Fernandez et al. 2012). Furthermore, psychrotolerant strains of Pseudomonas alleviated cold stress symptoms in wheat seedlings and induced chilling resistance in tomato by inducing the expression of the cold regulated transcription factors LeCBF1 and LeCBF3 (Subramanian et al. 2015). Our hypothesis is also supported by plant metagenome analysis, which showed a high proportion of bacterial genes present for tolerance against abiotic stress in the Sphagnum microbiome (Bragina et al. 2014).

Altogether, protection of plants against abiotic stress by bacteria opens the window for interesting biotechnological applications (Berg et al. 2016). Our study showed a novel example that metabolism and morphology of plants and their associated bacteria are intensively connected with each other, and an intense interplay of both maintains the functioning of the holobiont. Due to this importance, the microbiome should be considered in experimental botany.

\section{LITERATURE CITED}

Bai, Y., Müller, D. B., Srinivas, G., Garrido-Oter, R., Potthoff, E., Rott, M., Dombrowski, N., Münch, P. C., Spaepen, S., Remus-Emsermann, M., Hüttel, B., McHardy, A. C., Vorholt, J. A., and Schulze-Lefert, P. 2015. Functional overlap of the Arabidopsis leaf and root microbiota. Nature 528:364-369.

Berg, G., Köberl, M., Rybakova, D., Müller, H., Grosch, R., and Smalla, K. 2017. Plant microbial diversity is suggested as the key to future biocontrol and health trends. FEMS Microbiol. Ecol. 93(5).

Berg, G., Rybakova, D., Grube, M., and Köberl, M. 2016. The plant microbiome explored: Implications for experimental botany. J. Exp. Bot. 67:995-1002.

Berg, G., and Smalla, K. 2009. Plant species and soil type cooperatively shape the structure and function of microbial communities in the rhizosphere. FEMS Microbiol. Ecol. 68:1-13.

Bisht, S., Joshi, G., Haque, S., and Mishra, P. 2013. Cryotolerance strategies of Pseudomonads isolated from the rhizosphere of Himalayan plants. Springerplus 2:667.

Bodenhausen, N., Horton, M. H., and Bergelson, J. 2013. Bacterial communities associated with the leaves and the roots of Arabidopsis thaliana. PLoS One 8: e56329.

Bragina, A., Oberauner-Wappis, L., Zachow, C., Halwachs, B., Thallinger, G. G., Müller, H., and Berg, G. 2014. The Sphagnum microbiome supports bog ecosystem functioning under extreme conditions. Mol. Ecol. 23:4498-4510.

Bulgarelli, D., Rott, M., Schlaeppi, K., Loren van Themaat, E., Ahmadeinejad, N., Assenza, F., Rauf, P., Huettel, B., Reinhardt, R., Schmelzer, E., Peplies, J., Gloeckner, F. O., Amann, R., Eickhorst, T., and Schulze-Lefert, P. 2012. Revealing structure and assembly cues for Arabidopsis root-inhabiting bacterial microbiota. Nature 488:91-95.

Bulgarelli, D., Garrido-Oter, R., Münch, P. C., Weiman, A., Dröge, J., Pan, Y., McHardy, A. C., and Schulze-Lefert, P. 2015. Structure and function of the bacterial root microbiota in wild and domesticated barley. Cell Host Microbe 17:392-403.
Caporaso, J. G., Kuczynski, J., Stombaugh, J., Bittinger, K., Bushman, F. D., Costello, E. K., Fierer, N., Gonzalez Peña, A., Goodrich, J. K., Gordon, J. I., Huttley, G. A., Kelley, S. T., Knights, D., Koenig, J. E., Ley, R. E., Lozupone, C. A., McDonald, D., Muegge, B. D., Pirrung, M., Reeder, J., Sevinsky, J. R., Turnbaugh, P. J., Walters, W. A., Widmann, J., Yatsunenko, T., Zaneveld, J., and Knight, R. 2010. QIIME allows analysis of high-throughput community sequencing data. Nat. Methods 7:335-336.

Caporaso, J. G., Lauber, C. L., Walters, W. A., Berg-Lyons, D., Lozupone, C. A., Turnbaugh, P. J., Fierer, N., and Knight, R. 2011. Global patterns of $16 \mathrm{~S}$ rRNA diversity at a depth of millions of sequences per sample. Proc. Natl. Acad. Sci. USA 108(suppl.):4516-4522.

Cardinale, M., Grube, M., Erlacher, A., Quehenberger, J., and Berg, G. 2014. Bacterial networks and co-occurrence relationships in the lettuce root microbiota. Environ. Microbiol. 17:239-252.

Castrillo, G., Teixeira, P. J., Paredes, S. H., Law, T. F., and de Lorenzo, L. 2017. Root microbiota drive direct integration of phosphate stress and immunity. Nature 543:513-518.

Degenkolbe, T. 2012. Differential remodeling of the lipidome during cold acclimation in natural accessions of Arabidopsis thaliana. Plant J. 72: 972-982.

Fernandez, O., Theocharis, A., Bordiec, S., Feil, R., Jacquens, L., Clement, C., Fontaine, F., and Ait Barka, E. 2012. Burkholderia phytofirmans PsJN acclimates grapevine to cold by modulating carbohydrate metabolism. Mol. Plant-Microbe Interact. 25:496-504.

Gurian-Sherman, D., Lindow, S. E., and Panopoulos, N. J. 1993. Isolation and characterization of hydroxylamine-induced mutations in the Erwinia herbicola ice nucleation gene that selectively reduce warm temperature ice nucleation activity. Mol. Microbiol. 9:383-391.

Guy, C., Kaplan, F., Kopka, J., Selbig, J., and Hincha, D. K. 2008. Metabolomics of temperature stress. Physiol. Plant. 132:220-235.

Hardoim, P. R., van Overbeek, L. S., Berg, G., Pirttila, A. M., Compant, S., Campisano, A., Doring, M., and Sessitsch, A. 2015. The hidden world within plants: Ecological and evolutionary considerations for defining functioning of microbial endophytes. Microbiol. Mol. Biol. Rev. 79:293-320.

Hartmann, A., Rothballer, M., and Schmid, M. 2007. Lorenz Hiltner, a pioneer in rhizosphere microbial ecology and soil bacteriology research. Plant Soil 312:7-14.

Hildebrand, F., Tadeo, R., Voigt, A., Bork, P., and Raes, J. 2014. LotuS: An efficient and user-friendly OTU processing pipeline. Microbiome 2:30.

Hincha, D. K., Espinoza, C., and Zuther, E. 2012. Transcriptomic and metabolomic approaches to the analysis of plant freezing tolerance and cold acclimation. Pages 255-287 in: Improving Crop Resistance to Abiotic Stress. Wiley-Blackwell, Berlin.

Korn, M., Peterek, S., Mock, H.-P., Heyer, A. G., and Hincha, D. K. 2008. Heterosis in the freezing tolerance, and sugar and flavonoid contents of crosses between Arabidopsis thaliana accessions of widely varying freezing tolerance. Plant Cell Environ. 31:813-827.

Laforest-Lapointe, I., Paquette, A., Messier, C., and Kembel, S. W. 2017. Leaf bacterial diversity mediates plant diversity and ecosystem function relationships. Nature 546:145-147.

Leveau, J. H. J., and Lindow, S. E. 2001. Appetite of an epiphyte: Quantitative monitoring of bacterial sugar consumption in the phyllosphere. Proc. Natl. Acad. Sci. USA 98:3446-3453.

Lindow, S. E., and Brandl, M. T. 2003. Microbiology of the phyllosphere. J. Appl. Environ. Microbiol 69:1875-1883.

Love, M. I., Huber, W., and Anders, S. 2014. Moderated estimation of fold change and dispersion for RNA-seq data with DESeq2. Genome Biol. 15:550.

Lundberg, D. S., Yourstone, S., Mieczkowski, P., Jones, C. D., and Dangl, J. L. 2013. Practical innovations for high-throughput amplicon sequencing. Nat. Methods 10:999-1002.

Margesin, R. 2011. Psychrophilic microorganisms in alpine soils. Pages 187-198 in: Plants in Alpine Regions. Springer-Verlag, Vienna.

Masella, A. P., Bartram, A. K., Truszkowski, J. M., Brown, D. G., and Neufeld, J. D. 2012. PANDAseq: Paired-end assembler for illumina sequences. BMC Bioinformatics 13:31.

Mckhann, H. I., Gery, C., Bérard, A., Lévêque, S., Zuther, E., Hincha, D. I., De Mita, S., Brunel, D., and Teoule, E. 2008. Natural variation in CBF gene sequence, gene expression and freezing tolerance in the Versailles core collection of Arabidopsis thaliana. BMC Plant Biol. 8:105.

McMurdie, P. J., and Holmes, S. 2013. phyloseq: An R package for reproducible interactive analysis and graphics of microbiome census data. PLoS One 8: e61217.

McMurdie, P. J., and Holmes, S. 2014. Shiny-phyloseq: Web application for interactive microbiome analysis with provenance tracking. Bioinformatics 31 : 282-283. 
Mendes, R., Kruijt, M., de Bruijn, I., Dekkers, E., van der Voort, M., Schneider, J. H. M., Piceno, Y. M., DeSantis, T. Z., Andersen, G. L., Bakker, P. A. H. M., and Raaijmakers, J. M. 2011. Deciphering the rhizosphere microbiome for disease-suppressive bacteria. Science 332:1097-1100.

Mercier, J., and Lindow, S. E. 2000. Role of leaf surface sugars in colonization of plants by bacterial epiphytes. J. Appl. Environ. Microbiol. 66:369-374

Miller, W. G., Brandl, M. T., Quinones, B., and Lindow, S. E. 2001. Biological sensor for sucrose availability: Relative sensitivities of various reporter genes. J. Appl. Environ. Microbiol. 67:1308-1317.

Ottesen, A. R., Pena, A. G., White, J. R., Pettengill, J. B., Li, C., Allard, S., Rideout, S., Allard, M., Hill, T., Evans, P., Strain, E., Musser, S., Knight, R., and Brown, E. 2013. Baseline survey of the anatomical microbial ecology of an important food plant: Solanum lycopersicum (tomato). BMC Microbiol. 13:114.

Panke-Buisse, K., Lee, S., and Kao-Kniffin, J. 2017. Cultivated sub-populations of soil microbiomes retain early flowering plant trait. Microbiol. Ecol. 73: 394-403.

Panke-Buisse, K., Poole, A. C., Goodrich, J. K., Ley, R. E., and Kao-Kniffin, J. 2014. Selection on soil microbiomes reveals reproducible impacts on plant function. ISME J. 9:980-989.

Partida-Martínez, L. P., and Heil, M. 2011. The microbe-free plant: Fact or artifact? Front. Plant Sci. 2:100.

Peñuelas, J., Farre-Armengol, G., Llusia, J., Gargallo-Garriga, A., Rico, L., Sardans, J., Terradas, J., and Filella, I. 2014. Removal of floral microbiota reduces floral terpene emissions. Sci. Rep. 4:6727.

R Development Core Team. 2011. R: A language and environment for statistical computing. R Foundation for Statistical Computing, Vienna, Austria. http:// www.R-project.org

Rohde, P., Hincha, D. K., and Heyer, A. G. 2004. Heterosis in the freezing tolerance of crosses between two Arabidopsis thaliana accessions (Columbia-0 and C24) that show differences in non-acclimated and acclimated freezing tolerance. Plant J. 38:790-799.

Rolli, E., Marasco, R., Vigani, G., Ettoumi, B., Mapelli, F., Deangelis, M. L., Gandolfi, C., Casati, E., Previtali, F., Gerbino, R., Cei, F. P., Borin, S., Sorlini, C., Zocchi, G., and Daffonchio, D. 2015. Improved plant resistance to drought is promoted by the root-associated microbiome as a water stress-dependent trait. Environ. Microbiol. 17:316-331.

Santos-Medellín, C., Edwards, J., Liechty, Z., Nguyen, B., and Sundaresan, B. 2017. Drought stress results in a compartment-specific restructuring of the rice root-associated microbiomes. MBio 8:e00764-17.

Schlaeppi, K., Dombrowski, N., Oter, R. G., Themaat, E. V. L. V., and SchulzeLefert, P. 2014. Quantitative divergence of the bacterial root microbiota in Arabidopsis thaliana relatives. Proc. Natl. Acad. Sci. USA 111:585-592.

Schmidt, R., Koberl, M., Mostafa, A., Ramadan, E. M., Monschein, M., Jensen, K. B., Bauer, R., and Berg, G. 2014. Effects of bacterial inoculants on the indigenous microbiome and secondary metabolites of chamomile plants. Front. Microbiol. 5:64.

Schmieder, R., and Edwards, R. 2011. Quality control and preprocessing of metagenomic datasets. Bioinformatics 27:863-864.
Schulz, E., Tohge, T., Zuther, E., Fernie, A. R., and Hincha, D. K. 2015. Natural variation in flavonol and anthocyanin metabolism during cold acclimation in Arabidopsis thaliana accessions. Plant Cell Environ. 38:1658-1672.

Smallwood, M., and Bowles, D. J. 2002. Plants in a cold climate. Philos. Trans. R. Soc. Lond. B Biol. Sci. 357:831-847.

Steponkus, P. L. 1984. Role of the plasma membrane in freezing injury and cold acclimation. Annu. Rev. Plant Physiol. 35:543-584.

Subramanian, P., Mageswari, A., Kim, K., Lee, Y., and Sa, T. 2015. Psychrotolerant endophytic Pseudomonas sp. strains OB155 and OS261 induced chilling resistance in tomato plants (Solanum lycopersicum Mill.) by activation of their antioxidant capacity. Mol. Plant-Microbe Interact. 28: 1073-1081.

Thorsen, J., Brejnrod, A., Mortensen, M., Rasmussen, M. A., Stokholm, J., Al-Soud, W. A., Sorensen, S., Bisgaard, H., and Waage, J. 2016. Large-scale benchmarking reveals false discoveries and count transformation sensitivity in 16S rRNA gene amplicon data analysis methods used in microbiome studies. Microbiome 4:62.

Turner, T. R., James, E. K., and Poole, P. S. 2013. The plant microbiome. Genome Biol. 14:209.

Verginer, M., Siegmund, B., Cardinale, M., Muller, H., Choi, Y., Miguez, C. B., Leitner, E., and Berg, G. 2010. Monitoring the plant epiphyte Methylobacterium extorquens DSM 21961 by real-time PCR and its influence on the strawberry flavor. FEMS Microbiol. Ecol. 74:136-145.

Vorholt, J. A. 2012. Microbial life in the phyllosphere. Nat. Rev. Microbiol. 10: 828-840.

Wagner, M. R., Lundberg, D. S., Coleman-Derr, D., Tringe, S. G., Dangl, J. L., and Mitchell-Olds, T. 2014. Natural soil microbes alter flowering phenology and the intensity of selection on flowering time in a wild Arabidopsis relative. Ecol. Lett. 17:717-726.

Weigel, D. 2012. Natural variation in Arabidopsis: From molecular genetics to ecological genomics. Plant Physiol. 158:2-22.

Weiss, S., Xu, Z. Z., Peddada, S., Amir, A., Bittinger, K., Gonzalez, A., Lozupone, C., Zaneveld, J. R., Vázquez-Baeza, Y., Birmingham, A., Hyde, E. R., and Knight, R. 2017. Normalization and microbial differential abundance strategies depend upon data characteristics. Microbiome 5:27.

Weller, D. M., Raaijmakers, J. M., Gardener, B. B. M., and Thomashow, L. S. 2002. Microbial populations responsible for specific soil suppressiveness to plant pathogens. Annu. Rev. Phytopathol. 40:309-348.

Wilson, A. C., and Duncan, R. P. 2015. Signatures of host/symbiont genome coevolution in insect nutritional endosymbioses. Proc. Natl. Acad. Sci. USA 112:10255-10261.

Xin, Z., and Browse, J. 2000. Cold comfort farm: The acclimation of plants to freezing temperatures. Plant Cell Environ. 23:893-902.

Yang, J., Kloepper, J. W., and Ryu, C.-M. 2009. Rhizosphere bacteria help plants tolerate abiotic stress. Trends Plant Sci. 14:1-4.

Zhen, Y., and Ungerer, M. C. 2008. Clinal variation in freezing tolerance among natural accessions of Arabidopsis thaliana. New Phytol. 117:419-427.

Zuther, E., Schulz, E., Childs, L. H., and Hincha, D. K. 2012. Clinal variation in the non-acclimated and cold-acclimated freezing tolerance of Arabidopsis thaliana accessions. Plant Cell Environ. 35:1860-1878. 